\title{
Two are better than one: noninvasive assessment of liver fibrosis in nonalcoholic fatty liver disease
}

\author{
Grace Lai-Hung Wong $\cdot$ Henry Lik-Yuen Chan
}

Received: 13 January 2015/Accepted: 27 February 2015/Published online: 11 March 2015

(C) Asian Pacific Association for the Study of the Liver 2015

Nonalcoholic fatty liver disease (NAFLD) is currently the most common cause of chronic liver disease in many regions, affecting $15-40 \%$ of the general population [1,2]. One of the key challenges in managing NAFLD is differentiating simple steatosis from advanced disease, i.e., those with nonalcoholic steatohepatitis (NASH), advanced fibrosis or even cirrhosis [3]. The challenge is further substantiated by the poor correlation between liver fibrosis and routine blood tests, e.g., the serum alanine aminotransferase (ALT) level [4]. Patients may have progressed to advanced fibrosis or cirrhosis without any abnormalities in these common blood parameters [5]. Liver biopsy is not always feasible or acceptable to patients, especially to those largely asymptomatic subjects; this has promoted the popularity of noninvasive assessments of liver fibrosis for NAFLD patients (Table 1) [6, 7].

Among the numerous noninvasive assessments, liver stiffness measurement (LSM) by transient elastography is one of the most popular tools [8]. This tool out-performed other serum parameters in detecting advanced fibrosis or cirrhosis [9]. Nonetheless, a false-positive diagnosis of advanced fibrosis is not uncommon, in addition to LSM results lying in the gray zone [9]. This would be an even

G. L.-H. Wong · H. L.-Y. Chan

Institute of Digestive Disease, The Chinese University of Hong

Kong, Shatin, Hong Kong

G. L.-H. Wong · H. L.-Y. Chan $(\bowtie)$

Department of Medicine and Therapeutics, The Chinese

University of Hong Kong, 9/F Prince of Wales Hospital, 30-32

Ngan Shing Street, Shatin, Hong Kong

e-mail: hlychan@cuhk.edu.hk

G. L.-H. Wong · H. L.-Y. Chan

State Key Laboratory of Digestive Disease, The Chinese

University of Hong Kong, Shatin, Hong Kong more serious problem in obviously obese subjects, as the LSM is higher among those with a body mass index (BMI) above $30 \mathrm{~kg} / \mathrm{m}^{2}$ in the same fibrosis stage [10]. On the other hand, the NAFLD fibrosis score (NFS) is the beststudied serum-based combined clinical algorithm; it was recommended in the latest American practice guidelines for NAFLD [11]. All these observations have raised the interest in combining these two well-studied noninvasive parameters. On the other hand, magnetic resonance elastography (MRE) is probably even more accurate than LSM and NFS [12]. Nonetheless, MRE is not as widely available as these two tools.

Recently, Chan et al. [13] evaluated the diagnostic performance a novel two-step approach combining NFS and LSM. The beauty of this study was the robust methodology of including two independent groups of patients as the training and validation cohort, respectively. The detailed comparison of the performance of various approaches, be it either parameter alone or combining two parameters in different fashions, established this novel twostep approach as the best among the five to avoid liver biopsy. Similar approaches have been proposed in patients with chronic hepatitis B $[14,15]$; this was the first time in NALFD patients.

The unresolved questions remaining include the suboptimal performance of NFS in Asian subjects because of the different BMI cutoffs for obesity [16]. This was echoed by the relatively low sensitivity (40\%) of NFS to predict advanced fibrosis in Chan's study. Investigators should pay special attention when applying any parameters derived from the west to Asian patients, such that different cutoffs or even different algorithms should be considered. It would be helpful if another serum parameter with better performance could be found. Cytokeratin-18 (CK18) fragments have been investigated extensively as novel biomarkers for 
Table 1 Noninvasive parameters for advanced fibrosis in nonalcoholic fatty liver disease [6, 9, 12]

\begin{tabular}{|c|c|c|c|c|}
\hline Parameters & Cutoffs & Components & Sensitivity & Specificity \\
\hline \multirow{2}{*}{$\begin{array}{l}\text { Liver stiffness } \\
\text { measurement }(\mathrm{kPa})\end{array}$} & 7.9 & \multirow[t]{2}{*}{ N.A. } & 0.77 & 0.69 \\
\hline & 9.6 & & 0.64 & 0.84 \\
\hline NAFLD fibrosis score & -1.455 and 0.676 & $\begin{array}{l}\text { Age, hyperglycemia, BMI, platelet, } \\
\text { albumin, AST/ALT ratio (dual cutoffs) }\end{array}$ & 0.77 & 0.96 \\
\hline BARD score & 2 & BMI, AST/ALT ratio, diabetes & 0.62 & 0.66 \\
\hline AST/ALT ratio & 1.0 & AST, ALT & 0.21 & 0.90 \\
\hline APRI & 0.5 and 1.5 & AST, platelets (dual cutoffs) & 0.65 & 0.97 \\
\hline ELF & 0.3576 & Hyaluronic acid, TIMP1, PIIINP (dual cutoffs) & 0.80 & 0.90 \\
\hline FIB-4 & 1.30 and 2.67 & Age, AST, platelet, ALT (dual cutoffs) & 0.74 & 0.98 \\
\hline FibroTest & 0.70 & $\begin{array}{l}\text { Total bilirubin, GGT, } \alpha_{2} \text {-macroglobulin, } \\
\text { ApoA1, haptoglobin (dual cutoffs) }\end{array}$ & 0.88 & 0.99 \\
\hline MRE (kPa) & 3.63 & N.A. & 0.86 & 0.91 \\
\hline C-caffeine breath test ${ }^{\mathrm{a}}$ & 1.27 & N.A. & 0.90 & 0.76 \\
\hline
\end{tabular}

$A L T$ alanine aminotransferase, ApoA1 apolipoprotein A1, APRI AST-to-platelet ratio index, AST aspartate aminotransferase, $B M I$ body mass index, ELF enhanced liver fibrosis panel, GGT gamma-glutamyl transpeptidase, MRE magnetic resonance elastography, NAFLD nonalcoholic fatty liver disease, PIIINP procollagen III amino-terminal peptide, TIMP1 tissue inhibitor of matrix metalloproteinase 1

${ }^{a}$ The cutoff of 1.27 on the C-caffeine breath test was used to diagnose histological cirrhosis

NASH [17, 18], but in the latest guideline it was considered premature to recommend them in routine clinical practice [11].

Another big area for further investigation is the prospective longitudinal assessment of liver fibrosis in NAFLD subjects. Luckily a grossly elevated ALT level is uncommonly seen in NAFLD subjects such that the confounding effect of ALT on LSM [19] would be minimal. The current major limitation of all these noninvasive tools is that they have largely been investigated in cross-sectional studies; thus, their utility in monitoring a disease's natural history and predicting outcomes or responses to therapeutic interventions remains to be defined. Since liver fibrosis changes relatively slowly compared to liver fat, with short-term lifestyle modifications [20], studies of sufficiently long follow-up duration would be prudent to demonstrate the change in liver fibrosis [21]. Recently, NFS was found to be valuable in predicting liver-related events and deaths in a study with a median follow-up duration of nearly 9 years [22]. More data from similar studies would be useful to ascertain the role of these noninvasive tools.

A newly published phase 2 study by the NASH Clinical Research Network tested the use of obeticholic acid, a farnesoid X receptor agonist, in NASH (the FLINT trial) and first demonstrated that a pharmacological agent improved liver fibrosis after 18 months of treatment. This exciting observation not only shed the light on the therapeutic aspect of NASH, it also shows the need for noninvasive tools to assess the on-treatment response of liver fibrosis [23].
In conclusion, the study by Chan et al. provides valuable data on this novel two-step approach combining NFS and LSM to improve the accuracy of predicting advanced fibrosis and reducing the need for liver biopsy. Future study should be directed toward the values of these tools in a longitudinal fashion.

Compliance with ethical requirements and Conflict of interest All procedures followed were in accordance with the ethical standards of the responsible committee on human experimentation (institutional and national) and with the Helsinki Declaration of 1975, as revised in 2008. Informed consent was obtained from all patients for being included in the study. Grace Wong has received honoraria for lectures for Echosens and Furui. Henry Chan is a consultant for Furui and has received an honorarium for a lecture for Echosens.

\section{References}

1. Williams CD, Stengel J, Asike MI, Torres DM, Shaw J, Contreras $\mathrm{M}$, et al. Prevalence of nonalcoholic fatty liver disease and nonalcoholic steatohepatitis among a largely middle-aged population utilizing ultrasound and liver biopsy: a prospective study. Gastroenterology 2011;140:124-131

2. Wong VW, Chu WC, Wong GL, Chan RS, Chim AM, Ong A, et al. Prevalence of non-alcoholic fatty liver disease and advanced fibrosis in Hong Kong Chinese: a population study using proton-magnetic resonance spectroscopy and transient elastography. Gut 2012;61:409-415

3. Chan HL, de Silva HJ, Leung NW, Lim SG, Farrell GC. AsiaPacific working party on N. How should we manage patients with non-alcoholic fatty liver disease in 2007? J Gastroenterol Hepatol 2007;22:801-808

4. Wong VW, Wong GL, Tsang SW, Hui AY, Chan AW, Choi PC, et al. Metabolic and histological features of non-alcoholic fatty 
liver disease patients with different serum alanine aminotransferase levels. Aliment Pharmacol Ther 2009;29:387-396

5. Wong VW, Wong GL, Choi PC, Chan AW, Li MK, Chan HY, et al. Disease progression of non-alcoholic fatty liver disease: a prospective study with paired liver biopsies at 3 years. Gut 2010;59:969-974

6. Kwok R, Tse YK, Wong GL, Ha Y, Lee AU, Ngu MC, et al. Systematic review with meta-analysis: non-invasive assessment of non-alcoholic fatty liver disease-the role of transient elastography and plasma cytokeratin-18 fragments. Aliment Pharmacol Ther 2014;39:254-269

7. Musso G, Gambino R, Cassader M, Pagano G. Meta-analysis: natural history of non-alcoholic fatty liver disease (NAFLD) and diagnostic accuracy of non-invasive tests for liver disease severity. Ann Med 2011;43:617-649

8. Wong GL. Transient elastography: Kill two birds with one stone? World J Hepatol 2013;5:264-274

9. Wong VW, Vergniol J, Wong GL, Foucher J, Chan HL, Le Bail $\mathrm{B}$, et al. Diagnosis of fibrosis and cirrhosis using liver stiffness measurement in nonalcoholic fatty liver disease. Hepatology 2010;51:454-462

10. Wong GL, Chan HL, Choi PC, Chan AW, Lo AO, Chim AM, et al. Association between anthropometric parameters and measurements of liver stiffness by transient elastography. Clin Gastroenterol Hepatol 2013;11:295-302, e291-e293

11. Chalasani N, Younossi Z, Lavine JE, Diehl AM, Brunt EM, Cusi $\mathrm{K}$, et al. The diagnosis and management of non-alcoholic fatty liver disease: practice guideline by the American Association for the Study of Liver Diseases, American College of Gastroenterology, and the American Gastroenterological Association. Hepatology 2012;55:2005-2023

12. Loomba R, Wolfson T, Ang B, Hooker J, Behling C, Peterson M, et al. Magnetic resonance elastography predicts advanced fibrosis in patients with nonalcoholic fatty liver disease: a prospective study. Hepatology 2014;60:1920-1928

13. Chan WK, Nik Mustapha NR, Mahadeva S. A novel 2-step approach combining the NAFLD fibrosis score and liver stiffness measurement for predicting advanced fibrosis. Hepatol Int 2015. doi:10.1007/s12072-014-9596-7

14. Wong GL, Wong VW, Choi PC, Chan AW, Chan HL. Development of a non-invasive algorithm with transient elastography
(Fibroscan) and serum test formula for advanced liver fibrosis in chronic hepatitis B. Aliment Pharmacol Ther 2010;31:1095-1103

15. Wong GL, Chan HL, Choi PC, Chan AW, Yu Z, Lai JW, et al. Non-invasive algorithm of enhanced liver fibrosis and liver stiffness measurement with transient elastography for advanced liver fibrosis in chronic hepatitis B. Aliment Pharmacol Ther 2014;39:197-208

16. Wong VW, Wong GL, Chim AM, Tse AM, Tsang SW, Hui AY, et al. Validation of the NAFLD fibrosis score in a Chinese population with low prevalence of advanced fibrosis. Am J Gastroenterol 2008;103:1682-1688

17. Shen J, Chan HL, Wong GL, Choi PC, Chan AW, Chan HY, et al. Non-invasive diagnosis of non-alcoholic steatohepatitis by combined serum biomarkers. J Hepatol 2012;56:1363-1370

18. Dvorak K, Stritesky J, Petrtyl J, Vitek L, Sroubkova R, Lenicek $\mathrm{M}$, et al. Use of non-invasive parameters of non-alcoholic steatohepatitis and liver fibrosis in daily practice - an exploratory case-control study. PLoS One 2014;9:e111551

19. Wong GL, Wong VW, Choi PC, Chan AW, Chim AM, Yiu KK, et al. Increased liver stiffness measurement by transient elastography in severe acute exacerbation of chronic hepatitis B. J Gastroenterol Hepatol 2009;24:1002-1007

20. Wong VW, Chan RS, Wong GL, Cheung BH, Chu WC, Yeung $\mathrm{DK}$, et al. Community-based lifestyle modification programme for non-alcoholic fatty liver disease: a randomized controlled trial. J Hepatol 2013;59:536-542

21. Wong GL, Chan HL, Yu Z, Chan AW, Choi PC, Chim AM, et al. Coincidental metabolic syndrome increases the risk of liver fibrosis progression in patients with chronic hepatitis $\mathrm{B}-\mathrm{a}$ prospective cohort study with paired transient elastography examinations. Aliment Pharmacol Ther 2014;39:883-893

22. Angulo P, Bugianesi E, Bjornsson ES, Charatcharoenwitthaya $\mathrm{P}$, Mills PR, Barrera F, et al. Simple noninvasive systems predict long-term outcomes of patients with nonalcoholic fatty liver disease. Gastroenterology 2013;145:782-789, e784

23. Neuschwander-Tetri BA, Loomba R, Sanyal AJ, Lavine JE, Van Natta ML, Abdelmalek MF, et al. Farnesoid X nuclear receptor ligand obeticholic acid for non-cirrhotic, non-alcoholic steatohepatitis (FLINT): a multicentre, randomised, placebo-controlled trial. Lancet 2014. doi:10.1016/S0140-6736(14)61933-4 